\title{
A Low Complexity Subspace-Based Blind Channel Estimation For MIMO-OFDM
}

\author{
Zhang Yankui ${ }^{1, \mathrm{a}}$, Wang Daming ${ }^{2, \mathrm{~b}}$, Chen Song ${ }^{3, \mathrm{c}}$ \\ 1,2,3The PLA Information Engineering University,Zhengzhou City, 450001, China \\ a834799435@qq.com, bwdm123 @aliyun.com, cwirelessmancs@163.com
}

Keywords: OFDM; permutation and combination; subspace method; blind channel estimation

\begin{abstract}
The current subspace-based blind channel estimation for MIMO-OFDM system has the problems of high complexity due to matrix decomposition. In this paper, we proposed an improved subspace-based channel estimation algorithm, which is based on the method of the permutations and combinations on the transceiver signal. To reduce the complexity of singular value decomposition, this article put forward the idea of iterative instead. Theoretical analysis and simulation results showed that this algorithm could decrease the complexity compared with the traditional subspace algorithms in ensuring the accuracy and the converge.
\end{abstract}

\section{Introduction}

Orthogonal frequency division multiplexing (OFDM) is a popular transmission technique in wireless communication systems due to its advantages of high transmitted data rate and high spectrum efficiency. In an MIMO-OFDM system, channel estimation is the base of the signal synchronization and coherent demodulation, and also an important factor in determining the accuracy, sensitivity, and capacity of the system. In current, the channel estimation method for MIMO-OFDM system is mainly concentrated in two areas: First is the pilot-based estimation method. The main idea of these methods is that the sender transmits a pilot or training sequence, the receiver estimate the channel matrix based on the received pilot or training sequence. The advantage of these methods is that the estimated channel matrix is more accurate, and can estimate continuously. The typical methods are LS, MMSE and LMMSE estimation algorithm; the second is blind channel estimation method for MIMO-OFDM system. The main idea of these methods is based on some characteristics of the transmission signal itself, they do not need any pilot or training sequence. A typical blind channel estimation method is subspace-based blind channel estimation or STC(Space-Time Coding)-based method, these methods can greatly reduce the spectrum, and thus become the focus of current research in the field of channel estimation.

In the year of 2003, Roy.S, the $\mathrm{PhD}$ of the University of California gives an estimation method based on the virtual subcarriers of the OFDM system[1], the method has strong robustness, it can be used in the no cyclic prefix system, but its computational complexity is too high; Borching $\mathrm{Su}$, gives a new blind channel estimation algorithm based on noise subspace, compared to the traditional subspace algorithm, the new algorithm has greatly improved the accuracy and convergence, but this algorithm is only used for the SISO-OFDM system, the robustness is poor[3]. Kim .J .G et al proposed a blind channel estimation method based on the cyclic prefix of the signal, for an improved method without the need for continuous excitation. It can achieve better performance, but this method was applied only in SISO-OFDM system[6]. There are some subspace-based blind channel estimation algorithms based on reorganization of the time-domain signal, these methods can achieve better system accuracy and perform with fast convergence, but have high complexity[2,4,5].

In summary, the subspace-based blind channel estimation methods have become the main methods of MIMO-OFDM system channel estimation with saving resource conservation and having high accuracy without sending and receiving pilots. However, the existing subspace-based blind channel estimation algorithm have the advantages of slow convergence, high complexity and poor robustness, 
this paper presents a quick convergence of low complexity channel estimation algorithm to overcome these.

\section{Subspace-Based Blind Estimation Model}

MIMO-OFDM system combines the advantages of the technoleges of MIMO and OFDM,its model as Fig.1:
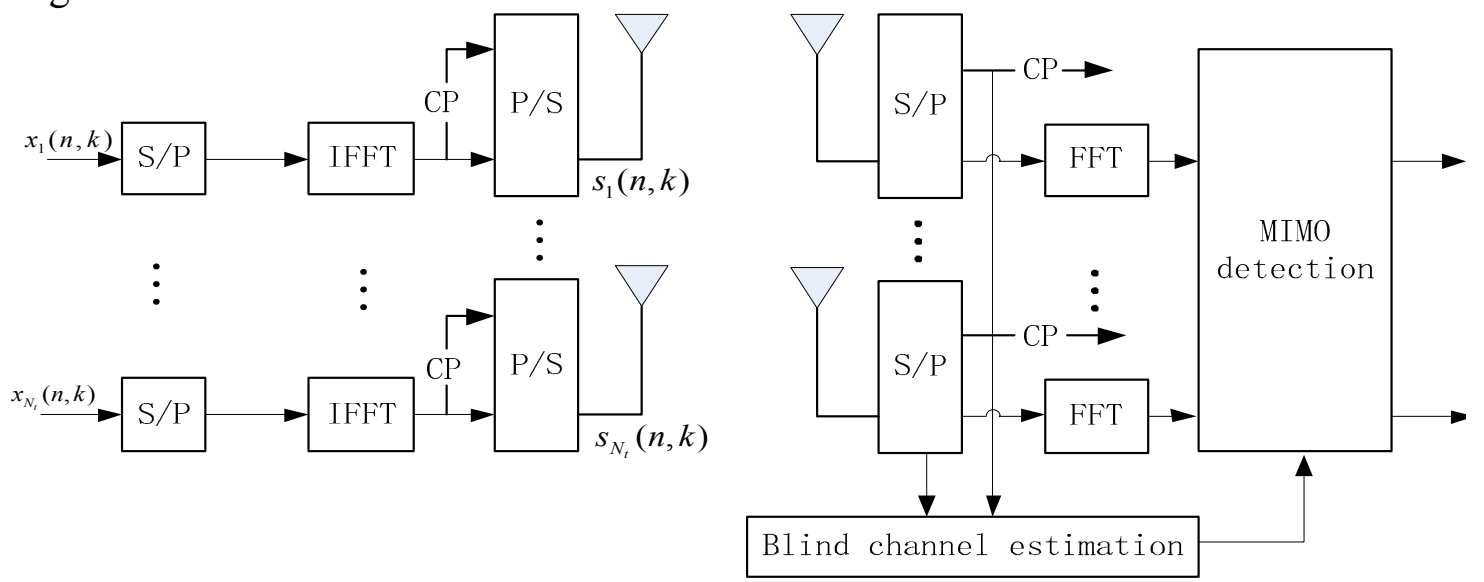

Fig. 1 The Model of MIMO-OFDM system

Suppose that there are $N_{t}$ transmitting antennas, and $N_{r}$ receiving antenna, FFT points is $M$, the length of circle prefix is $P$, OFDM symbol length is $Q=M+P, x_{i}(n, k)$ represents the $k t h$ carrier of the $n t h$ in the $i t h$ receiving antenna. The data before the modulation of OFDM data can be expressed as

$$
x(n, k)=\left[\begin{array}{c}
x_{1}(n, k) \\
x_{2}(n, k) \\
\vdots \\
x_{N_{t}}(n, k)
\end{array}\right]
$$

The $n$th OFDM signal of all transmission antennas is represented as

$$
x(n)=\left[\begin{array}{c}
x\left(n, k_{0}\right) \\
x\left(n, k_{0}+1\right) \\
\vdots \\
x\left(n, k_{0}+D-1\right)
\end{array}\right]
$$

After the modulation, the OFDM data is represented as

$$
s(n, k)=\left[\begin{array}{c}
s_{1}(n, k) \\
s_{2}(n, k) \\
\vdots \\
s_{N_{t}}(n, k)
\end{array}\right]
$$

The $n t h$ transmision OFDM signal is expressed as: 


$$
s(n)=\left[\begin{array}{c}
s(n, M-P) \\
\vdots \\
s(n, M-1) \\
s(n, 0) \\
\vdots \\
s(n, M-1)
\end{array}\right]
$$

The corresponding received signal at the receiving can be expressed

$$
y(n)=\left[\begin{array}{c}
y_{c p}(n) \\
y_{M}(n)
\end{array}\right]=\left[\begin{array}{c}
y(n, 0) \\
\vdots \\
y(n, Q-1)
\end{array}\right]
$$

Among them

$$
\begin{gathered}
y_{M}(n)=\left[\begin{array}{c}
y(n, P) \\
\vdots \\
y(n, Q-1)
\end{array}\right] \\
y(n, k)=\left[\begin{array}{c}
y_{1}(n, k) \\
y_{2}(n, k) \\
\vdots \\
y_{N_{t}}(n, k)
\end{array}\right]
\end{gathered}
$$

Since the tap coefficient of the lth path for channel matrix is expressed as

$$
h(l)=\left[\begin{array}{cccc}
h_{11}(l) & h_{12}(l) & \cdots & h_{1 N_{t}}(l) \\
h_{21}(l) & h_{22}(l) & \cdots & h_{2 N_{t}}(l) \\
\vdots & \vdots & \vdots & \vdots \\
h_{N_{r} 1}(l) & h_{N_{r} 2}(l) & \cdots & h_{N_{r} N_{t}}(l)
\end{array}\right]
$$

Which $l=0,1,2 \cdots L, L \leq P$, in order to facilitate the calculation, it may be $L=P$, so the channel matrix being estimated is represented as

$$
h=[h(0), h(1), \cdots h(L)]
$$

Define the channel matrix, which was expressed as follows:

$$
H_{0}=\left[\begin{array}{cccccc}
h(L) & \cdots & h(0) & 0 & \cdots & 0 \\
0 & h(L) & \cdots & h(0) & \cdots & 0 \\
\vdots & \vdots & \ddots & \ddots & \ddots & \vdots \\
0 & 0 & \cdots & h(L) & \cdots & h(0)
\end{array}\right]
$$

We extend the channel matrix with the type of Toeplitz, $m$ is defined as the Toeplitz matrix expansion coefficient

$$
\tilde{H}=\left[\begin{array}{ccc}
H_{0} & \cdots & 0 \\
\vdots & \ddots & \vdots \\
0 & \cdots & H_{0}
\end{array}\right]
$$


Suppose consecutively received $m$ OFDM symbols, which was expressed as follows:

$$
\left[\begin{array}{c}
y_{M}(0) \\
y_{M}(1) \\
\vdots \\
y_{M}(m-1)
\end{array}\right]=\left[\begin{array}{ccc}
H_{0} & \cdots & 0 \\
\vdots & \ddots & \vdots \\
0 & \cdots & H_{0}
\end{array}\right]_{M N_{r} m \times Q N_{t} m}\left[\begin{array}{c}
s(0) \\
s(1) \\
\vdots \\
s(m-1)
\end{array}\right]+\left[\begin{array}{c}
\eta(0) \\
\eta(1) \\
\vdots \\
\eta(m-1)
\end{array}\right]
$$

permutations and combinations for the OFDM symbols in the receiver and sender signals, we can achieve the relationship of (13):

$$
\begin{gathered}
{\left[\begin{array}{ccc}
y_{M}(0) & \cdots & y_{M}(m-1) \\
y_{M}(1) & \cdots & y_{M}(m-2) \\
\vdots & \cdots & \vdots \\
y_{M}(m-1) & \cdots & y_{M}(0)
\end{array}\right]_{M N_{r} m \times m !}=\tilde{H}_{M N_{r} m \times Q N_{t} m}\left[\begin{array}{ccc}
s(0) & \cdots & s(m-1) \\
s(1) & \cdots & s(m-2) \\
\vdots & \cdots & \cdots \\
s(m-1) \cdots & s(0)
\end{array}\right]_{Q N_{t} m \times m !}} \\
+\left[\begin{array}{ccc}
\eta(0) & \cdots & \eta(m-1) \\
\eta(1) & \cdots & \eta(m-2) \\
\vdots & \cdots & \vdots \\
\eta(m-1) & \cdots & \eta(0)
\end{array}\right]
\end{gathered}
$$

It can be seen from (13), through permutations and combinations, the transmission signal rank changes from 1 to $m$ !, and the rank of the receiver matrix increase $m m$ ! every time we receive an OFDM signal, that is, when the received continuous incentived for an OFDM symbol is $J$, there are $A_{J}^{m}$ unrelated columns vector in the matrix $S$.

The dimension of channel matrix $H$ is $M N_{r} m \times Q N_{t} m$, if we want to identify the modulated signal $s$, a necessary requirement as follow

$$
Q N_{t} m \leq A_{J}^{m}
$$

$J$ is the number of persistent excitation, and $J \geq m$. If the expansion coefficient of the channel matrix is 1 , then the algorithm rollback to the traditional space algorithm.

Subspace-based channel estimation is based on the SVD decomposition for the received signal autocorrelation matrix to obtain the signal subspace and noise subspace, $V$ is constructed by the noise subspace matrix, with the orthogonality between the signal subspace and noise subspace,we construct the cost function below:

$$
\hat{h}=\underset{\|h\|_{F}=1}{\arg \min }\|h V\|^{2}=\underset{\|h\|_{F}=1}{\arg \min } h^{H} V V^{H} h
$$

We will get the final estimation of the channel matrix based on the cost function.

\section{Fast Blind Channel Estimation Of Low Complexity}

Based on the analysis above, this subspace based channel estimation algorithm can convergence qukickly,but it is too complex. The complexity of the traditional subspace methods focused on the SVD decomposition of the received signal, and showed an increase $O\left(n^{3}\right)$, this paper increase the received signal for $m$ times, and which in engineering practice is unacceptable, especially when $m$ is large. To overcome this disadvantage, this paper first use $\mathrm{QR}$ decomposition algorithm to get the signal subspac, and then finish the search of noise subspace using Smith of Manchester orthogonal, last complete blind channel estimation,therefor this algorithm avoid the high complexity caused by the SVD decomposition. 
First, assuming $\Phi(n)$ have no change over time in the whole process, then carry out QR decomposition for $A(n)$

$$
\begin{aligned}
& A(n)=Q(n) R(n) \\
& A(n)=\Phi(n) Q(n-1)
\end{aligned}
$$

Because of the updating of self-correlation matrix as follow

$$
R_{Y}(n)=\alpha R_{Y}(n-1)+(1-\alpha) y(n) y^{H}(n)
$$

$\alpha$ is the forgetting factor, characterizing the weigth of the new auto-correlation matrix in the original autocorrelation matrix. Replace $\Phi(n)$ with $R_{Y}(n)$, then we will get the following expression

$$
A(n)=\alpha A(n-1)+(1-\alpha) y(n)\left(Q^{H}(n-1) y(n)\right)^{H}
$$

According to the formula (19), if $A(n-1), y(n), Q(n-1)$ are known, we can update and get $A(n)$, and then obtain $Q(n)$ from (16), because of $Q(n)$ finally converge to the signal subspace $U_{s}$. We will get the noise subspace $U_{n}$ according to Smith of Manchester orthogonal.From the whole process, the idea of an iterative replaces the complex matrix decomposition, thereby reducing the complexity of the algorithm.

Because the signal subspace of the received signal correlation matrix $R_{Y}$ is equal to the space spanned with the column vector of $H$, so

$$
U_{n}^{H} \tilde{H}=0
$$

Since $\tilde{H}$ is the block-Toeplitz matrix, and its upper-left corner of the first block matrix is $H_{0}$, then the above equation is equal to

$$
U_{n}^{H} H_{0}=0
$$

The dimension of $U_{n}$ is $m M N r \times m N r(M-D)$, its column vector span the noise subspace.

Defined vector $u_{k}$, the $k$ th column vector of $U_{n}$, change it as follows:

$$
u_{k}=\left[v_{k, 1}^{T}, v_{k, 2}^{T}, \cdots v_{k, m M}{ }^{T}\right]^{T}
$$

$v_{k, i}{ }^{T}$ is a column vector of $N r \times 1$.

Structure matrix

$$
V_{k}=\left[\begin{array}{cccccc}
v_{k, 1} & \cdots & v_{k, m M} & 0 & \cdots & 0 \\
0 & v_{k, 1} & \cdots & v_{k, m M} & \cdots & \vdots \\
\vdots & \vdots & \ddots & \ddots & \ddots & 0 \\
0 & \cdots & 0 & v_{k, 1} & \cdots & v_{k, m M}
\end{array}\right]
$$

Obviously $u_{k} h$ is equal to $V_{k} h$, so that:

$$
V=\left[V_{1}, V_{2} \cdots V_{m N r(M-D)}\right]
$$

Establish a cost equation of channel estimation matrix is:

$$
\hat{h}=\underset{\|h\|_{F}=1}{\arg \min }\|h V\|^{2}=\underset{\|h\|_{F}=1}{\arg \min } h^{H} V V^{H} h
$$

$\|\cdot\|_{F}$ represents Frobenius norm. Channel matrix $h$ is the eigenvectors of the $N_{t}$ minimum corresponding eigenvalues corresponding of $V V^{H}$ eigendecomposed. The estimated value of the channel matrix exists a fuzzy matrix with the real channel matrix, in practice it can be eliminated by a small number of pilot.

The main steps of the algorithm are summarized as follows: 
1)To make the received signal unrelated,we do some trade-offs based on the OFDM signal. Using (11) to expand the channel matrix, expansion coefficient is $m$;

2)Make corresponding permutations and combinations of the signal in the sending and receiving as the form of formula (13), the speed of a matrix rank increase from 1 to $m$ !.

3) Judging it satisfy $Q N_{t} m \leq A_{J}^{m}$ or not, if not meet, then continued accumulation of OFDM symbols until it meet;

4) Iteration the signal subspace with $Q R$ decomposition, and then calculate the noise subspace based on the orthogonality between the signal subspace and the noise subspace with Schmit;

5) Construct the cost function and solve the channel matrix based on the formula (25)

\section{Simulation And Performance Analysis}

In order to validate the performance of the algorithm, the paper make some simulation on the effectiveness of the algorithm and convergence speed, simulation environment is as follows: set the number of transmission antennas $N_{t}=2$, receiving antennas $N_{\mathrm{r}}=2$, FFT points $M=32$, the lengths of CP is $P=8$, the length of OFDM symbol $Q=32$, channel order $L=P=8$, the signal use QPSK modulation. Situation in all simulations, assuming that the system had been completed accurately signal synchronization, $m=1$ represent the performance simulation curve of the original subspace algorithm

Simulation 1: the NMSE of the different expansion coefficients of the channel matrix in the same received data block.

In this simulation, Fig. 3 shows the estimation results of the normalized mean square error with the curve of the SNR. Among them, the normalized mean square error calculation formula (26) as follows:

$$
N M S E=\sqrt{\frac{1}{N_{m}\|h\|_{2}^{2}} \sum_{j=1}^{N_{m}}\left\|h^{(j)}-\hat{h}^{(j)}\right\|}
$$

Among them, $N_{m}$ is the number of simulation, this simulation takes $N_{m}=50$, the number of received data blocks $J=20$, channel matrix expansion coefficients were taken: $m=1,4,7,10,15,20$ the simulation results have been shown in Fig. 3:

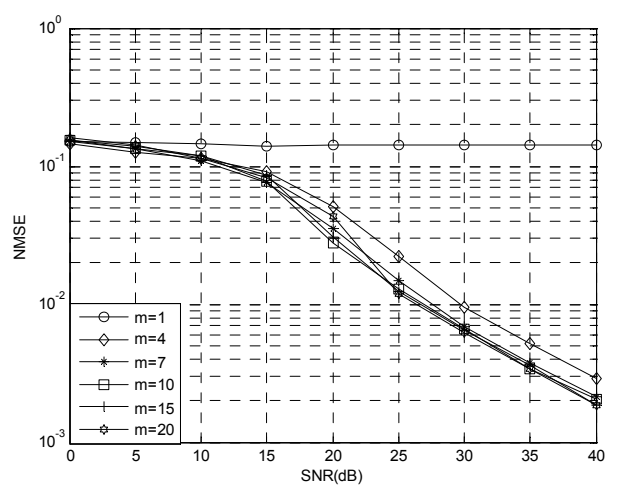

Fig.2 NMSE change with SNR of different expansion coefficients of the channel matrix

Fig. 2 shows that, $m=1$ have a poor performance or failed in a relatively small amount of data, it is because the number of the received data block is too small that cannot meet the convergence condition of the original algorithm; $m \geq 2$ represents convergence speed curve of the algorithm, it can be seen from the Fig.3, when the amount of data is small, this paper can achieve the estimation results and fast convergence by increasing the channel matrix expansion coefficients.And the bigger the channel matrix expansion coefficient, the higher utilization of information, and thus the faster convergence speed. But when expansion coefficient increases to a certain extent, NMSE decline with SNR advantage is not obvious, then the estimated result of the algorithm to stabilize.

Simulation 2: the comparison of NMSE before and after the simplification 
In this simulation, set the channel matrix expansion coefficient $m=5$, the number of received data blocks from 0 to 100, the number of simulations $N_{m}=50$, NMSE change with $J$ curve in Fig. 3:

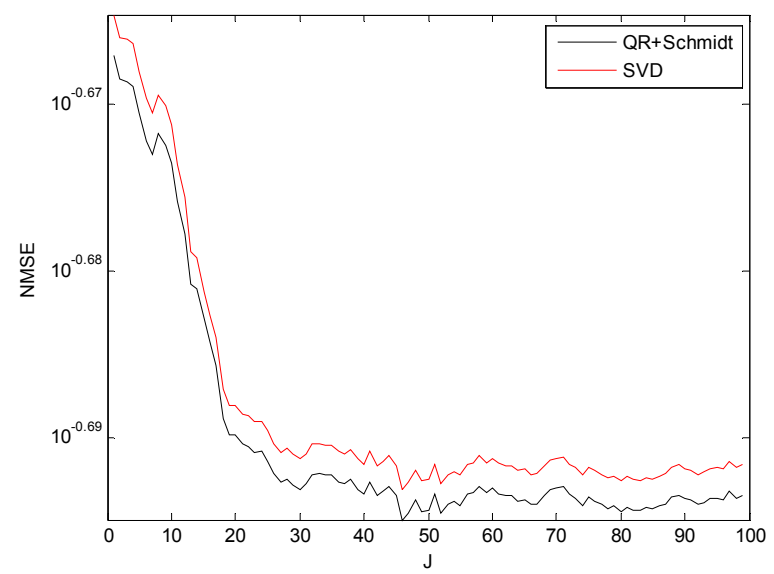

Fig. 3 the comparison of NMSE before and after the simplification

Fig.3 simulation results show that when the received data block is small, the simplification algorithm perform poor, channel estimation fails. With the increase in the number of the received data block, the simplification algorithm is better than the original SVD method; when the number of the received data block increases to a certain amount, the performance improves basically stabilized,so with the same condition,QR and Schmidt perform better than SVD.

\section{Summary}

Acconding to the analysis of the MIMO-OFDM system model, we improved a fast blind channel estimation algorithm based on permutations and combinations, which convergence quickly, but also a corresponding increase in computational complexity, to reduce the complexity of singular value decomposition,this article put forward the idea of iterative instead.Theoretical analysis and simulation results show that, compared with the traditional subspace algorithms, this algorithm have a strong advantage in complexity under the same convergence speed.

\section{References}

[1] Li C,Roy S.Subspace-based blind channel estimation for OFDM by exploiting virtual carriers[J].IEEE Trans Wireless Commun,2003, 2(1):141-150.

[2] P. Loubaton and E. Moulines, "On blind multiuser forward link channel estimation by the subspace method: Identifiability results,'IEEE Trans. Signal Process., vol. 48, no. 8, pp. 2366-2376,Aug. 2000.

[3] C. Shin, R. W. Heath and E. J. Power, Blind channel estimation for MIMO-0FDM systems [J].IEEE Trans.Veh.Technol,Mar.2007, 56(2):670-680.

[4] T. P. Krauss and M. D. Zoltowski, "Bilinear approach to multiuser second-order statistics-based blind channel estimation,” IEEE Trans. Signal Process., vol. 48, no. 9, pp. 2473-2486, Sep. 2000.

[5] Fang S H, Chen J Y, Shieh M D, et al. Modified Subspace Based Channel Estimation Algorithm for OFDM Systems.[C]// Vehicular Technology Conference, 2009. VTC Spring 2009. IEEE 69th. IEEE, 2009:1 - 5.

[6] Kim J G, Oh J H, Lim J T. Subspace-Based Channel Estimation for MIMO-OFDM Systems With Few Received Blocks[J]. IEEE Signal Processing Letters, 2012, 19(7):435 - 438.

[7] Fang S H, Chen J Y, Lin J S, et al. Blind channel estimation for MIMO-OFDM systems with repeated time-domain symbols[C]// Circuits and Systems (APCCAS), 2012 IEEE Asia Pacific 
Conference on. IEEE, 2012:37 - 40

[8]Tu C C, Champagne B. Subspace-Based Blind Channel Estimation for MIMO-OFDM Systems With Reduced Time Averaging[J]. IEEE Transactions on Vehicular Technology, 2010, 59(3):1539 1544.

[9] Y. Hua and J. K. Tugnait, "Blind identifiability of FIR-MIMO systems with colored input using second order statistics,” IEEE Signal Process.Lett., vol. 7, no. 12, pp. 348-350, Dec. 2000. 